\title{
Attitudes et représentations déclarées vis -à -vis du français hors du cadre universitaire Approche sociolinguistique
}

\section{Dr. Racha Mohamed Mahmoud ${ }^{*}$}

rmm02@fayoum.edu.eg

\section{Résumé}

Cette étude vise à étudier les attitudes et les représentations de l'usage du français dans un contexte hors du cadre universitaire à l'aide d'un questionnaire comportant trois axes: situation linguistique et niveau de maîtrise du français, contact et conflit des langues, examiner directement les attitudes. Ce questionnaire est proposé à un échantillon d'étudiants (50), à majorité féminin, exclusivement francophones et natifs du Fayoum. L'analyse statistique des données chiffrées permet d'analyser certains phénomènes sociolinguistiques comme la relation entre le type d'attitude et les variables sociales, les motivations de choisir le français comme code de communication, l'alternance codique et ses fonctions communicationnelle et identitaire, sécurité et insécurité linguistique et identité linguistique et sociale. Les étudiants sont également invités à la fin du questionnaire à fournir un exemple de leur propre pratique du français. L'analyse de ces productions montre un soin accordé à l'éthos.

Mots clés : Attitude, (in)sécurité linguistique, alternance codique, identité linguistique, forme normative, contact/conflit des langues.

"Le simple fait de communiquer par le langage engage toute la personne, c'est-à- dire un individu avec ses expériences antérieures, son adhésion à des croyances et des valeurs culturelles et intellectuelles"(Martinez, 2011: 9)

\section{* Université du Fayoum}

(Attitudes et représentations déclarées..)Dr. Racha Mohamed Mahmoud 


\section{$\underline{\text { Introduction }}$}

Le langage est un fait social qui engendre diverses attitudes en fonction des paramètres de la situation d'échange. Dans cette étude, on cherche à étudier les représentations, les attitudes et les motivations de l'usage du français dans un contexte hors du cadre universitaire (entre les membres de la famille, les amis, les camarades,...) et non pas dans un contexte professionnel (les études). En fait, hors du contexte scolaire, le degré de formalité et les exigences normatives sont faibles. L'attitude, est "l'acte conscient, savoir-faire enseignable, qui permet à un sujet locuteur de choisir une langue face à une situation donnée, mais aussi d'entendre d'autres langues et de s'y intéresser, de connaitre et d'utiliser des procédés d'ajustements linguistiques"(Rispail, 2006:88). Selon le Dictionnaire de linguistique et des sciences du langage, l'attitude se définit comme " l'ensemble des opinions explicites ou implicites sur l'usage d'une langue"(. Dubois et al., 2012: 57). D'après D. Lafontaine, "le terme d'attitude linguistique est employé parallèlement, et sans véritable nuance de sens, à représentation, norme subjective, évaluation subjective, jugement, opinion"(1997: 56-57). W. Labov le définit ainsi : "un système de réactions subjectives régulières", "caractéristiques des locuteurs en fonction de leur appartenance sociale". Il est évident que l'attitude change d'un lieu à l'autre, d'un individu à l'autre et chez un même individu, d'une situation à l'autre en fonction de certains paramètres social, culturel et identitaire.

En effet, il n'y a pas de rapport neutre entre le locuteur et sa langue. Mais, " il existe (...) tout un ensemble d'attitudes, de sentiments des locuteurs face aux langues, aux variétés de langues et à ceux qui les utilisent, qui rendent superficielle l'analyse de la langue comme un simple instrument"(Calvet, 2003: 46). En

(Attitudes et représentations déclarées..)Dr. Racha Mohamed Mahmoud 
général, le locuteur manifeste diverses attitudes envers la langue qu'il utilise ou apprend: positive/négative, acceptation/refus, maintien/changement, inclusion/exclusion, etc. L' attitude nous permet de dégager les spécificités de leur parler bi/plurilingue et les divers moyens pour parvenir à aménager le contact de ces langues, de s'interroger sur la présence des paramètres relevant d'une linguistique, réellement inégalitaire et d'identifier le cadre socio-culturel et socio-psychologique qui sous-tend la pratique verbale. Autrement dit, l'attitude linguistique aide à saisir les sentiments et à observer les comportements des locuteurs envers une telle ou telle langue.

Quant à la notion de représentation, elle signifie l'activité mentale déterminant la position des individus par rapport à une langue, à une culture et à ses usagers; c'est "l'apparition de l'image verbale mentale chez le locuteur" (Dubois et al., 2012: 410). Il y a donc une correspondance entre attitude et représentation puisque toutes les deux informent sur les rapports entre langues et locuteurs et sur la manière dont elles influencent leur pratique langagière. D'ailleurs, elles se réfèrent à des images, des pensées et des sentiments envers telle langue.

Cette recherche est basée sur un questionnaire (voir annexe 1), élaboré par la chercheuse dans une finalité de bien cerner les attitudes envers le français, utilisé au quotidien. Il vise également à analyser les attitudes à propos des pratiques bi/plurilingues dans des situations de communication diverses. Nous essayons de dévoiler ce que les étudiants imaginent être ou plutôt l'être réellement. Autrement dit, nous cherchons à préciser le type d'attitudes (appréciation /dépréciation) et de stéréotypes à l'usage du français dans tel contexte et à saisir les valeurs "relevant de la sphère socio-affective (chaleur humaine, 
sympathie, solidarité, douceur,...)"(Lafontaine, 19997:58) qui y sont attachées.

Le questionnaire est proposé aux étudiants de la troisième année(2019), département de français, faculté des Lettres, université du Fayoum, à un public de (50) étudiants, à majorité féminin, exclusivement francophones et natifs du Fayoum. Le terrain universitaire, institution normative par excellence, est un lieu privilégié de divers contacts interpersonnel et linguistique, surtout à la faculté des Lettres où il y a différents départements : anglais, chinois, arabe, français, ...etc. Une différence est notable au niveau de leur milieu social, de leur niveau de français, de leur lieu de résidence. La passation du questionnaire se fait en présence de la chercheuse afin d'éclaircir certains termes paraissant ambigus pour les étudiants et de s'assurer qu'ils comprennent tout avant de répondre et d'être à leur disposition en cas de problème. Le questionnaire commence par un certain nombre de variables sociales: sexe, âge, lieu de résidence,...

Ce questionnaire comporte, à part les questions sur l'identité, trois axes visant à recueillir des informations sur le profil linguistique de ces étudiants et leur pratique langagière. Le premier porte sur leur situation linguistique et le niveau de leur maîtrise du français, le deuxième est focalisé sur le mélange des langues et le troisième concerne leurs comportements et représentations vis-à-vis du français. D'ailleurs, les étudiants ont le choix de présenter quelques exemples de leur pratique réelle du français à la fin du questionnaire. L'étude se fonde donc sur l'analyse des réponses au questionnaire et de leur pratique réelle $\mathrm{du}$ français apportée en complément à ces réponses. Les étudiants sont désignés, tout au long de la recherche par l'initiale "E", tandis que les questions sont notées par Q suivi du numéro en vue de leur ordre dans le questionnaire mis en place. Il est à noter 
que ce questionnaire vient à la suite d'un cursus de linguistique portant essentiellement sur les courants de la linguistique (structuralisme, fonctionnalisme, actes de parole et surtout sociolinguistique), d'où la clarté de certaines notions qui $\mathrm{y}$ figurent comme (in)sécurité linguistique, contact des langues, alternance codique, etc.

La situation linguistique de ces étudiants est assez complexe. Ils ont à leur disposition deux variétés de l'arabe (arabe standard et arabe dialectal) et à peu près deux variétés du français(le français standard, appris et développé dans un contexte scolaire et, pour ainsi dire, le français arabisé, ou le français à l'égyptienne(le faux français) où il y a des déficiences, dues au manque de compétences linguistiques. En plus, ils connaissent et utilisent l'anglais. Le français est essentiellement la langue des études. L'anglais, première langue apprise, est abandonné au bénéfice $\mathrm{du}$ français. L'arabe, correspondant à la dimension identitaire, langue maternelle, et langue parlée dans la rue, en famille, pourtant la variété adoptée (l'arabe dialectal) est loin de la variété normative qu'on apprend à l'école. Y a -t-il un passage d'une langue à l'autre, si oui, est -il en rapport avec le positionnement identitaire du locuteur? Dans cette pratique déclarée de trilingue, la préférence de l'une au profit des autres est-elle un choix personnel, relève-t-elle de la liberté linguistique? Etre plurilingue entraîne-t-il parfois que l'on plonge dans une situation schizophrénique? Leur pratique verbalise-t-elle une certaine sécurité ou insécurité linguistique? Est-elle la marque d'un travail conscient d'adhésion à la communauté francophone? La logique culturelle a-t-elle son mot à dire?

Ce groupe d'étudiants est défini en fonction de différentes variables sociales dans la mesure où la langue est conçue non seulement comme un système autonome et isolé mais plutôt en

$\overline{\text { (Attitudes et représentations déclarées..)Dr. Racha Mohamed Mahmoud }}$ 
rapport avec l'environnement social. Le critère de fréquence permet de calculer et de dénombrer les phénomènes compris dans le questionnaire tout en les croisant avec les variables sociales afin de bien étudier les attitudes et les comportements qui en découlent. Les variables de chaque question dénotent des attitudes différentes. Quelle attitude émerge -t- elle la plus? Les étudiants possèdent-ils une attitude positive vis-à-vis du français? Cette attitude relève $-\mathrm{t}$-elle de la rupture ou de l'association du langage des jeunes? Celle -ci correspond -elle à des fonctions discursives et communicationnelles diverses? Excluent ou incluent -elle certains aspects conflictuels? Reflète-t-elle certains comportements linguistiques des locuteurs francophones? La présence réelle ou plutôt potentielle de plusieurs langues dénotet-elle un véritable contact des langues : symétrique et égalitaire ou plutôt un faux contact? La question identitaire et la préservation de sa propre culture constituent -t- elles les mots d'ordre dans ce dispositif langagier? Quels enjeux et quelles démarches sont-ils mis en vigueur dans ce mixage linguistique? Sont-ils conscients de leur répertoire langagier? Leurs comportements discursifs suggèrent-ils certaines qualités et présentations de soi (ethos)?

Cette étude vise, dans un premier temps, à analyser statistiquement les données chiffrées de la réponse à ce questionnaire, à préciser, dans un deuxième temps, les attitudes de ces étudiants envers le français dans un contexte extracurriculaire et à montrer s'il y a une relation entre ces attitudes et les variables sociales testées dans le questionnaire. Elle a finalement pour finalité d'éclaircir certaines notions sociolinguistiques attachées à ces attitudes: contact/ conflit des langues, rapport langue/identité, alternance codique, sécurité/insécurité linguistique, choix approprié du code dans tel contexte.

$\overline{\text { (Attitudes et représentations déclarées..)Dr. Racha Mohamed Mahmoud }}$ 


\section{1-Analyse statistique des données}

\section{$\underline{\text { Axe } 1 \text { : Situation linguistique et niveau de maîtrise du }}$ francais}

Dans Q.1, 62\% préfèrent communiquer en français en zone francophone par rapport à $32 \%$ entre pairs et $6 \%$ entre les membres de famille. Le taux du français, Q.2, dans leur échange quotidien est moyen pour $86 \%$ des étudiants. La majorité (82\%) croit que la langue apprise en premier temps, l'anglais, paraît plus élaborée que le français et elle facilite même, selon 64\%, la communication en français, Q.3 et Q.4. Ce groupe d'étudiants considère donc la situation bilingue comme "une bonne chose et apporte du positif à la communauté. Le fait d'évoluer dans un milieu bilingue permet plus facilement l'acquisition d'une seconde langue"(Perret, 2016: 129). Ils (50\%) choisissent l'anglais comme langue étrangère dans laquelle ils se sentent à l'aise au milieu familial, Q.31.

Selon Q.5, 56\% d'étudiants pensent avoir un répertoire pauvre en français, et $38 \%$ ont l'impression de posséder un répertoire riche et complexe. Un grand nombre d'étudiants $(76 \%)$ est satisfait de ses compétences linguistiques, $4 \%$ seulement sousestiment leur pratique, Q.6. Dans Q.8, 56\% croient bien parler le français, contre $14 \%$ qui croient parler mal le français. Pourtant, un pourcentage de $30 \%$ estime parler le français à sa façon. A propos de l'écart écrit/oral, Q.7, 48\% affirment bien cet écart, tandis que $18 \%$ pensent que l'écrit est au service de l'oral.

Très souvent, nous notons qu'ils n'ont pas de véritable perception de la norme, à tel point que $52 \%$ d'entre eux ne savent pas s'ils envisagent des difficultés à se comprendre, $30 \%$ trouvent qu'ils ont des difficultés à ce propos et $18 \%$ seulement sont certains de la réussite de leur échange. Concernant, les activités

(Attitudes et représentations déclarées..)Dr. Racha Mohamed Mahmoud 
en français (Q.10, Q.11, Q.12): suivre les journaux en français, $74 \%$ les lisent parfois; écrire en français, $98 \%$ préfèrent écrire un conte, $2 \%$ optent pour la variable des articles et personne ne choisit l'écriture d'un roman; suivre les chaînes de la télé, $70 \%$ les fréquentent mais rarement.

Quant à leur communication en français, 68\% se dirigent vers un discours libre, non-formalisé qui ne suit pas les règles prescrites, Q.13. Leur pratique du français, Q.14, ne diffère pas d'une situation à l'autre. Elle semble être identique dans toutes les situations selon72\%. Lors de l'échange, ils envisagent des problèmes linguistiques variés, Q.15: choix du lexique approprié $(48 \%)$, structure grammaticale $(24 \%)$, et prononciation correcte (28\%). Leur système graphique, Q.16, est diversifié d'après $62 \%$. Cependant, $10 \%$ ont recours à des émoticônes. Leur emploi des rieurs, Q.17, est soit minoritaire (40\%), soit inexistant (56\%). Le degré de spontanéité, Q.19, se trouve limité pour $46 \%$, n'existe pas selon $42 \%$ et énorme pour $12 \%$ seulement. $50 \%$ jouent sur les sonorités et les schémas intonatifs, Q.20. $46 \%$ ont fréquemment recours à des énoncés normatifs, Q.21.

\section{$\underline{\text { Axe } 2 \text { : contact/conflit des langues }}$}

Si la communication en français, Q.22, s'avère impossible, $98 \%$ ont recours à d'autres langues. Ainsi, le mélange des langues, Q.23, semble être d'ordre acquisitionnel (68\%), communicationnel (30\%), et identitaire (2\%). Parler bi/plurilingue assure, pour $56 \%$, une meilleure condition de la réussite de l'échange mais cette réussite dépend avant tout des paramètres de l'acte de l'échange, Q.24. Le contact des langues, Q.25, est donc évident. Dans leur pratique, les énoncés métissés cherchent, d'après 52\% à légitimer une certaine pratique langagière, à montrer l'insécurité linguistique (32\%) et à affirmer une 
compétence bi/plurilingue (16\%). Les marques transcodiques, Q.27, concernent divers niveaux : syntaxique, phonologique et évidemment lexical. La communication en français, Q.28, est soit symétrique (parler français et l'autre répond en français), selon $36 \%$, soit mixte (parler français et l'autre répond en plusieurs langues), selon52\%. D'ailleurs, l'articulation français/arabe, Q.29, correspond à un jeu (34\%), à un conflit (10\%) et à d'autres phénomènes $(56 \%)$.

\section{$\underline{\text { Axe } 3 \text { : examiner directement les attitudes }}$}

En effet, le choix de la langue de communication, Q.32, est déterminé par certains préjugés linguistiques. Ainsi, leur comportement linguistique vis-à-vis du français, Q.33, est relativement modifié. Pourtant, la charge affective, Q.34, à l'égard $\mathrm{du}$ français est forte pour 64\%. Lorsqu'ils communiquent en français, ils se sentent fiers et émouvants, Q.35, intégrés dans une communauté francophone et démarqués du groupe, Q.45. Ils éprouvent plutôt de la sécurité (60\%), contre $10 \%$ qui ressentent de l'insécurité, Q.57. Cependant, ils jugent exagérée une attitude telle que "le français n'est pas une langue avec laquelle on passe une information mais plutôt une langue avec laquelle on nait", Q.50. En plus, l'emphase et l'exagération que leur interlocuteur affiche en parlant français, Q.53, les irrite à tel point que face à cette attitude d'emphase, Q.54, 46\% lui font la remarque(petit commentaire là-dessus). Cet échantillon d'étudiants considère sa communication en français de plus en plus active, Q.36. D'après $62 \%$, parler français est un parler valorisant, Q.37.

L'objectif de leur communication en français, Q38, se trouve varié: se faire des amis, accomplir des affaires, communiquer intra-famille. Ils communiquent également en français, Q.43, afin de témoigner de leur culture (62\%), faire vivre 
cette langue dans leur milieu (22\%) et garder le contact avec des francophones (10\%). Leur pratique, Q.44, est conçue comme une forte volonté d'améliorer le degré de spontanéité et se familiariser avec le français. Parler français, Q.55, est doté des traits sociaux (76\%), psychologiques (14\%) et neurologiques (10\%). 56\% considèrent que la pratique du français sert à promouvoir plus de respect d'autrui, Q.56. Les étudiants l'utilisent, Q.39, tout d'abord pour discuter des sujets personnels $(60 \%)$, puis, pour faire une plaisanterie $(28 \%)$ et finalement pour aborder des sujets polémiques (12\%). D'après ce questionnaire, le français, Q.40, est préférable dans un contexte ludique $(48 \%)$ et cryptique $(44 \%)$, c'est pourquoi les fragments de plaisanterie en français, Q.41, sont énormes pour $76 \%$. S'éloigner du français normé, Q.59, sert à se libérer de l'univers scolaire (52\%), à provoquer le rire $(34 \%)$ et à créer une rupture avec toute forme de règle (14\%).

En outre, dans leur production verbale, Q.42, l'insulte rituelle $(58 \%)$ et les pratiques ludiques $(38 \%)$ se manifestent. Les revendications identitaires, Q.58, émergent plutôt d'une manière implicite selon $56 \%$. Communiquer en français est donc un signe de l'appartenance à la communauté des jeunes (62\%) et à la communauté francophone (30\%), Q.60. Le taux du français, Q.46, dépend du degré de formalité, de la charge affective et du besoin communicatif. Ainsi, l'intercompréhension ou la réussite de la communication, Q.48, est revendiquée comme un besoin selon $52 \%$. D'ailleurs, le changement de l'interlocuteur, Q.47, impose, pour $74 \%$, un changement de la façon de parler. Les garanties de la réussite de l'acte de l'échange, Q.49, sont relatives. Leur motivation, Q.51, pour continuer à communiquer en français demeurent stable selon 66\%. Leur famille, Q.52, apparait motivée à leur pratique. 


\section{2- Divers phénomènes sociolinguistiques}

La passation et l'analyse statistique d'un questionnaire proposé à des étudiants possédant relativement certaines compétences linguistiques donnent à voir un certain nombre de phénomènes langagiers :

\section{1 Pourquoi ce code?}

Pourquoi des locuteurs, de même origine, dans un échange informel, optent ils pour une langue étrangère? En effet, le choix d'un code bien précis, le français dans ce contexte, "apporte un supplément de sens au-delà du contenu de ce qui est encodé"(Wald, 1997: 71). Ce choix est généralement en fonction de l'intention de communication et de l'environnement de l'interaction et ses finalités. Chaque individu possède un répertoire linguistique, composé parfois de plusieurs codes. Celui-ci est pourvu de fonctions dans la communication sociale en fonction du choix du locuteur. Ces fonctions sont d'ordre extralinguistique, elles relèvent plutôt "de la régulation du système social"(Ibid : 72).

En fait, le besoin communicatif et acquisitionnel du langage est primordial, il constitue même "un aspect crucial du processus de socialisation"(Auger, 1997:15), acquérir une langue a pour fonction de lui permettre de communiquer avec les autres membres de cette communauté linguistique. Nous remarquons, chez ces étudiants, une sorte de coïncidence entre compétence linguistique et compétence communicative. Ils prennent en conscience les normes sociolinguistiques qui influencent et contrôlent la pratique langagière. Les étudiants adoptent donc la forme linguistique appropriée à la situation de communication, ce qui prouve qu'il y a une forte relation entre langage et facteurs sociaux. Autrement dit, " il existe un rapport beaucoup plus

(Attitudes et représentations déclarées..)Dr. Racha Mohamed Mahmoud 
complexe entre l'usage langagier et le contexte social" (Thiam, 1997:33). Leur comportement verbal est également en fonction de leur interlocuteur : " il est donc normal qu'ils varient aussi leurs productions langagières en fonction $\mathrm{du}$ contexte extralinguistique"(Auger, 1997:16). D'ailleurs, une variation graphique est notable en fonction du degré de formalité.

Les personnes enquêtées qui se servent de cette langue cherchent à se distinguer des autres étudiants issues de la même classe sociale et appartenant à la même sphère universitaire. Ainsi, désirent-ils appartenir à un niveau social beaucoup plus élevé ou plutôt masquer leur milieu d'appartenance et faire semblant d'appartenir à une sphère élevée à l'aide de cette façon de parler. Autrement dit, ils aiment (in)consciemment être assimilés à une classe dont le français était déjà un trait particulier. L'option pour cette langue est donc une marque de prestige. Ce groupe d'étudiants est régi d'un sentiment d'insécurité; il a une certaine prédilection pour le français de peur d'être socialement déprécié dans la mesure où le fait de trouver quelques mots d'une langue étrangère dans leur discours ordinaire est devenu une exigence. Le français, en tant que langue de communication, est donc pourvu d'une valeur emblématique d'étiquette sociale. Il est à noter que depuis très longtemps, le français est doté d'un pouvoir symbolique. Alors, cet exercice langagier leur donne accès à un niveau social plus ou moins supérieur, leur permet de s'approprier d'une position plus prestigieuse.

L'insistance à maintenir le français dans leur échange quotidien pourrait être le résultat d'un certain engagement moral envers cette langue. Autrement dit, cet usage est pourvu d'une certaine orientation, d'une certaine obligation. Les étudiants veulent promouvoir et même imposer la pratique du français au 
Fayoum. Ils se présentent ainsi en tant que défenseurs de cette langue et non pas comme simples sujets parlants parleurs. La plupart s'accorde à inscrire une image valorisante du français, équivalente à une reconnaissance socio-professionnelle très estimable. Ils veulent diffuser davantage le français au profit de l'anglais malgré un moindre pessimisme quant à son avenir et un sentiment de menace de l'anglais. Ils réagissent, à la manière, contre la hiérarchisation des langues au Fayoum. Cette volonté affirme que ce groupe d'étudiants désire se définir en tant que nouvelle génération qui véhicule de nouvelles formes de pratiques, de nouvelles valeurs à la fois sociales, linguistiques et identitaires. Le choix d'une langue signifie l'appartenance/l'exclusion à /d' une communauté linguistique donnée.

Pourtant, la variation du taux d'intégration du français est due au fait que chaque locuteur ne possède pas le même répertoire verbal. C'est également en fonction des paramètres de la situation communicationnelle que le choix est opéré. Cependant, ceux qui montrent une compétence défaillante du français, cherchent à la compenser par le fait de s'attacher à une autre communauté sociolinguistique, en l'occurrence anglophone. C'est d'une manière ou d'une autre un moyen pour sauver leur face.

Insérer le français dans leur échange quotidien signifie qu'ils confèrent davantage des valeurs sociales et interactionnelles et un pouvoir symbolique à cette langue dans ce contexte et qu'ils cherchent à évaluer et à considérer favorablement cette possibilité de s'exprimer. Les attributs sociolinguistiques qui y sont accordés pèsent sur la prédilection pour une telle pratique langagière. Cette insertion s'avère importante pour une certaine image de soi puisque le français est chargé de prestige social. Dans cet échantillon, tout locuteur possède un répertoire verbal. Il 
y fait un choix approprié en fonction de la situation de communication et de sa représentation de ses interlocuteurs. L'analyse de différentes modalités de leur pratique langagière donne à voir la disjonction entre le français en tant que langue d'étude et langue de communication. Le français, dans une approche communicative, constitue un moyen visant à les intégrer dans la communauté francophone. En fait, "la capacité de communiquer implique non seulement une connaissance des règles de la morphosyntaxe d'une langue, mais également une compétence culturelle"(Fioux et Tirvassen, 1997: 48). Les différentes modalités communicatives montrent que les étudiants se situent sur une échelle variée par rapport à la norme et se familiarisent généralement avec les exigences socio-culturelles régissant l'usage d'une telle langue.

\section{$\underline{\text { 2.2 Quel contexte? }}$}

Les étudiants sont capables d'identifier le modèle normatif et prestigieux et ont une attitude favorable à son égard. Pourtant, ils ne l'utilisent pas dans les conversations ordinaires. D'ailleurs, son usage en dehors du contexte scolaire souligne le caractère inévitable de sa vitalité et de son caractère dynamique et vivant. Les étudiants s'en servent afin de produire un effet comique surtout lorsqu'il est question du jeu de mot visant à faire rire leur partenaire. Cette visée (plaisanter en français) pourrait être différemment interprétée: refléter une certaine habileté et maîtrise ou contrairement déformer une langue dont la pression normative, acquise par la scolarisation et l'instruction, les gênent. Tout dépend de la manière de concevoir cette langue. Leurs remarques montrent que leur pratique est souvent marquée par le rire, l'émotion et le plaisir en communiquant avec ceux qui possèdent à peu près la même expérience linguistique et le même parcours scolaire. Cette attitude affirme que le français n'est donc pas pris

$\overline{\text { (Attitudes et représentations déclarées..)Dr. Racha Mohamed Mahmoud }}$ 
pour une langue extérieure à soi-même. Son usage entre pairs montre une grande aisance linguistique.

Le français est beaucoup utilisé dans un contexte ludique. Cet usage est conçu comme un phénomène individuel. C'est l'occasion de se libérer des contraintes linguistiques, du cadre formel. La valeur cryptique de l'usage du français, testée dans Q.40, assure une sorte de sphère sécurisante à ses membres puisqu'ils partagent un ensemble d'objets incompréhensibles pour les non-membres, exclus de cette sphère. C'est alors signe d'inclusion ou d'exclusion, c'est une manière pour se démarquer d'eux ou d'assimiler à eux.

Le français est beaucoup employé dans les jeux et les causeries manquant de sérieux et il figure rarement dans des discussions polémiques, $12 \%$, c'est ainsi qu'il figure beaucoup dans les fragments de plaisanterie, $76 \%$, parce que dans ce type de discours, tout est permis et même la transgression à la norme en est un aspect. Pourtant, sa figuration dans les fragments de débat n'est pas remarquable. Ceci signifie que les étudiants ne sont pas capables de défendre leurs points de vue ni d'en donner des preuves à l'appui en français. Plusieurs indices font valoir la notion de jeu langagier, celle-ci autorise une certaine modalité de parler qui fait partie du langage des jeunes. Ce dernier "leur permet de se reconnaitre comme appartenant au même groupe avec le souhait de se rapprocher le plus possible"(Trehel et Blanchet, 2006:72 ).

\section{$\underline{2.3 \text { Alternance codique }}$}

Leur pratique langagière témoigne d'un mélange codique et plus précisément emploi alterné de l'arabe et du français dans le même énoncé. D'ailleurs, ils subissent l'influence de l'anglais. En effet, le recours à l'alternance codique(le passage d'une langue à

(Attitudes et représentations déclarées..)Dr. Racha Mohamed Mahmoud 
une autre) pourrait être un effet inconscient de la possession de plusieurs langues. Cependant ses déterminations et ses motivations conscientes sont évidentes : chercher à montrer qu'on maîtrise plusieurs langues, à s'amuser, dans un but ludique et à aménager les langues.

Ce phénomène assure des buts acquistionnels : le locuteur s'engage dans un processus d'acquisition afin de réaliser un certain progrès dans son apprentissage et des buts communicationnels : le locuteur cherche en premier lieu à garantir la réussite de son projet communicationnel. En principe, l'alternance codique est pris pour "un phénomène occasionnel, accidentiel ou idiosyncrasique: les occurrences d'alternance codique seraient fonction de préférences individuelles et leur applicabilité sociale serait imprévisible "(Thiam, 1997: 34) mais, il se manifeste en tant que signe de solidarité à un tel groupe.

La présence des énoncés alternés dans leur échange hors du cadre universitaire a également des buts identitaires, c'est indiquer une certaine appartenance communautaire. M. Rispail formule que " le recours à l'autre langue (aux autres langues) est une bouée, une façon de s'en sortir mieux(...) c'est un marquage de domaine, de territoire, de compétences, d'actions possibles" (2006: 87). De même, J. A. Lopez note que l'alternance codique relève d' "une alternance situationnelle qui marque une rupture /un rapprochement entre le locuteur et un ou une partie de ses interlocuteurs"(Lopez, 2006: 147) et qu'elle se présente "comme traduisant un "dialogue interne", signe d'un clivage identitaire du locuteur"(Ibid). D'après ce point de vue, ce phénomène reflète une certaine tension identitaire, une sorte de conflit d'identité. En d'autres termes, le locuteur se trouve tiraillé entre son appartenance à la communauté arabe et plus précisément égyptienne et son adoption des normes et valeurs françaises. 
Nous parvenons donc à affirmer qu'il "apparait alors comme un moyen de mettre en scène, plus ou moins consciemment (...) les différentes facettes de son personnage"( Ibid: 153).

Par ce procédé, ils mettent en place deux langues, deux identités, deux communautés. En gros, ils assurent une sorte de compromis à la fois langagière et identitaire. Alors, les deux langues se rencontrent et se mélangent, créant ainsi une pratique langagière libre et valorisant de nouvelles idées. Ce mélange paraît également comme une meilleure solution pour un état de tiraillement entre deux langues. Il protège le locuteur de deux sortes de peur : ne pas maîtriser d'emblée le français et perdre sa langue d'origine. Par ce faire, il croit être admis dans les deux sphères franco-arabes.

Le recours à des énoncés alternés témoignant donc de l'appartenance à une telle ou telle langue et correspondant à un certain contexte énonciatif relève de la pure volonté du locuteur d'opter pour cette pratique langagière qui gomme la frontière entre le français et l'arabe- malgré la divergence étymologique- à tel point que leur identification s'avère difficile : s'agit -il d'un français sur le modèle arabe ou au contraire un arabe sur le modèle français? A certaines reprises, nous avons l'impression que ce type de production langagière est une sorte de plaisir. Mélanger plusieurs langues paraît amusant pour eux. Le français et l'arabe ne se présentent pas, d'après la majorité de ce groupe d'étudiants, comme deux camps linguistiques et identitaires conflictuels. L'un n'exclut pas l'autre.

Ce phénomène est régi par certains paramètres : changement du degré de formalité/familiarité, changement du public, changement de type de discours privé/public. Ce passage interlangue s'articule sur divers plans : le lexique, la construction 
syntaxique et même la phonologie. Mais, en gros, les étudiants possèdent une représentation sociale positive des contacts de langues. Dans ce contexte trilingue, ils maintiennent leur langue maternelle. Ceci est dû à des facteurs externes: durée du séjour en Egypte(ne pas voyager ailleurs), âge (manque d'expériences) et les spécificités culturelles propres à cette langue. Cette attitude contredit donc l'opinion soutenant que "les situations de contacts de langues constituent l'un des principaux facteurs de perte de la première langue ou langue maternelle(L1) dans un environnement de langue seconde (L2)" (Akinci, 2006:129).

\section{$\underline{\text { 2. } 4 \text { Attitudes favorable/défavorable }}$}

Parler une langue, c'est en même temps parler de soimême, de son identité, de la place que cette langue a au sein d'un groupe social, de ses rapports avec des interlocuteurs qu'ils soient français ou arabe, d'où la variation des attitudes. M. Grawitz note qu' " avoir vis-à-vis de quelque chose, c'est donner un sens à ce quelque chose, le percevoir plus ou moins hostile ou sympathique"(Grawitz, 1996: 458). Alors, la manière de perception d'un tel ou tel phénomène est le critère de son acceptation ou de son rejet. Les attitudes s'organisent sur un axe vertical dont l'admiration et le rejet constituent ses deux pôles et entre lesquels il existe divers degrés d'attitudes. La manière dont le locuteur perçoit et évalue sa pratique langagière diffère d'une personne à l'autre, d'un moment à l'autre, et même chez la même personne, d'une situation à l'autre. Mais nous essayons de concevoir ses attitudes dans leur ensemble. Leur attitude, l'ensemble de jugements et de tendances, est généralement positive, 62\%, quel que soit leur profil socio-culturel. Il s'agit également d'une attitude de proximité en regard du français puisqu'ils l'emploient hors du contexte des études. Leur attitude correspond donc à un degré plus élevé et plus conscient de la

(Attitudes et représentations déclarées..)Dr. Racha Mohamed Mahmoud 
perception de leur usage tout en évitant toute sorte de difficulté c'est pourquoi $6 \%$ d'entre eux seulement ont du mal à s'évaluer, Q.5.

L'attitude négative, malgré son moindre pourcentage, 8 $\%$, désigne un rejet non seulement linguistique mais plutôt culturel et identitaire. L'attitude neutre $30 \%$ est considérée comme une mise à distance soignée et protectrice. D'ailleurs, le fait de choisir la variable : "d'autres réponses, pas de réponse" comme c'est le cas dans Q. 13: d'autres réponses : 12\%, Q.18 : pas de réponse : $18 \%$, Q.32: d'autres réponses: 14\%, Q.47: pas de réponse : 16\%, Q.50: d'autres réponses : 18\%, Q.56 : d'autres réponses : $40 \%$ ou même d'éviter de répondre à certaines réponses, Q.38 à titre d'exemple, pourrait être interprété différemment:

- cette attitude constitue l'un des mécanismes de défense adopté par cette tranche afin de ne pas déclarer ouvertement leurs attitudes linguistiques, surtout négatives;

- elle montre la gêne éprouvée à l'égard de certaines questions; -elle reflète la difficulté à apporter une réponse catégorique et l'incertitude de leur positionnement et leur définition identitaire.

Ce groupe d'étudiants utilise le français, et l'évalue tout en ayant un certain nombre d'attitudes à son égard et à ceux qui le parlent en fonction du degré de conscience/inconscience de la présence d'une norme à la fois linguistique et sociale.

\subsubsection{Attitude /variables sociales}

Certaines variables sociales paraissent beaucoup plus significatives que d'autres, comme le sexe. Selon cette variable, un degré plus élevé d'insécurité linguistique et d'alternance 
codique est dénoté chez les filles par rapport aux garçons. Il est à rappeler qu'il s'agit d'un échantillon à majorité féminin. En effet, les filles prêtent beaucoup d'attention à leur façon de parler. Elles cherchent même à adopter un modèle beaucoup plus soigné. Celui-ci constitue une sorte de "pouvoir" et d'enjeu social qui assurent une image valorisante d'elles-mêmes.

Le clivage enseignement public/privé nous renseigne sur la catégorie sociale d'appartenance. Nous remarquons qu'accéder à l'université et avoir le statut d'étudiant leur confèrent une position beaucoup plus élevée quel que soit leur milieu social. Pourtant, les catégories assez inférieures montrent une attitude divergente. Certains affichent une attitude défavorable en regard du français, d'autres possèdent plutôt une attitude valorisante comme si le français garantissait une certaine ascension sociale selon leur point de vue. Pour la classe sociale assez aisée, nous enregistrons une corrélation entre le niveau social d'appartenance et des attitudes positives envers le français.

Quant à la variable du mode de vie urbain/rural, en général, la pratique langagière des locuteurs issus du milieu rural diffère de ceux issus du milieu urbain. Pourtant, cette distinction n'est pas nette dans le cas de notre étude parce que le Fayoum est à majorité ville rurale, il s'organise selon le système d'une ville agricole. L'usage d'une langue étrangère lors d'un échange quotidien soulève souvent une forme de critique, à valeur même "خواجه" [mutafarnis] ou simplement " منفرنس" d'insulte telle que [gawaja]. Alors, les valeurs identitaires et communautaires prônent sur cette pratique. Cependant, ce comportement linguistique change d'une famille à l'autre et d'une personne à l'autre. 
En gros, les étudiants ont des représentations sociolinguistiques positives quant au français. Ceci se lit dans sa fréquence dans leur pratique communicationnelle quotidienne. En plus, ils n'ont pas honte de le parler : 8\% seulement à la Q. 35, optent pour la réponse (honteux et dégradant) contre $58 \%$ pour la réponse (fier et émouvant). Ce type d'attitude est à mettre en rapport avec leur âge: ils sont encore jeunes, ils ne sont pas encore affrontés à des échecs sociolinguistiques. Les réponses avancées par les garçons et les filles ne sont pas tout à fait différentes. Ce résultat est dû à la ressemblance de leurs compétences linguistiques. De plus, le degré de familiarité avec l'allocutaire influence leur façon de parler. En tout cas, ils font preuve d'une certaine compétence linguistique même si celle-ci se trouve lacunaire. Leurs réactions quant à ce questionnaire nous informent sur l'avenir de cette langue. Ces attitudes favorables constituent un facteur qui les encourage à parler français entre eux et/ou avec autrui. Leur attitude dénote même un certain engagement à l'égard de cette langue

\subsection{Identité linguistique et sociale}

La langue et l'identité sont fortement liées et interdépendantes. La langue est porteuse d'identité, la définition identitaire est une partie intégrante de la conception linguistique. Ainsi, l'acquisition ou simplement l'usage d'une langue va de pair avec ceux de la reconnaissance de soi. En d'autres termes, l'identité se construit à travers la langue et celle-ci est signe de la dimension identitaire. Toute définition ou revendication identitaire se fait à l'aide de la langue et de même les modifications linguistiques se font, la plupart du temps, pour des raisons identitaires. 
Les étudiants veulent valoir leur identité sociolinguistique et avoir un certain type de rapport avec l'interlocuteur (supériorité/ infériorité ou égalité). Nous constatons qu'il s'agit :

- d'identité individuelle qui met le point sur des traits individuels, en l'occurrence, le fait d'être bilingue ou trilingue.

- d'identité communautaire, c'est le fait d'appartenir à une communauté bien précise en fonction de l'âge (communauté des jeunes: leur échange ,58\%, est doté des termes relevant du registre d'insulte, c'est l'un des traits de ce parler : Q.42) et de la langue soit maternelle (communauté arabe), soit étrangère (communauté francophone). Q.45: 56\% valorisent l'appartenance à cette communauté, Q.58, les revendications identitaires se manifestent clairement $20 \%$ et implicitement $56 \%$ et dans la Q. 60 l'appartenance communautaire est situé au premier plan.

Alors, l'acte langagier est en rapport étroit avec l'identité que les locuteurs désirent projeter. En d'autres termes, l'identité, quelle que soit son type, se marque à travers la pratique langagière. La langue assure donc un rôle unificateur. Pratiquer et communiquer en français pourrait être interprétée comme une preuve de vouloir appartenir à une telle communauté sociolinguistique tandis que les locuteurs qui abandonnent le français dans leurs échanges quotidiens semblent vouloir prendre une certaine distance et par suite refléter un désir de s'exclure de cette communauté. Cette attitude (s'inclure/s'exclure) est évidemment dictée par leur idéologie et leur identité. La pratique langagière équivaut donc à une recherche de se distinguer, de se comparer ou de se ressembler. Alors, leur usage s'attache à une certaine représentation dans leur imaginaire.

(Attitudes et représentations déclarées..)Dr. Racha Mohamed Mahmoud 
En effet, les locuteurs se trouvent dans une situation assez contradictoire. Ils désirent évidemment être reconnus comme francophones et en même temps conserver leur propre identité arabe. Ils se soucient de l'image que l'on projette d'eux. Dans leur échange, la langue arabe, porteuse de leur origine de leur identité rencontre la langue de l'autre. Cette rencontre permet de préserver l'être et l'autre, de promouvoir et en même temps de se définir. Ce rapport bilingue se voit donc en termes de complémentarité et de maniement linguistique. Ils essayent d'être arabes d'esprit français. Le français constitue leur propre outil pour se sentir intégré au groupe francophone, Q.45 et Q.60, et par suite accepté par lui.

Cependant, cet aspect n'empêche pas une certaine hésitation, malgré les attitudes positives, à utiliser le français dans ce contexte de peur de la perte de leur identité linguistique originale pour quelques personnes. Bien qu'ils étudient et maîtrisent relativement le français, il demeure une langue étrangère, une langue de l'Autre par opposition à celle du Même (la langue maternelle, l'arabe). En d'autres termes, pour cette minorité $2 \%$ : Q.23, le français n'est pas considéré comme la langue légitime qu'ils doivent utiliser dans ce contexte. Légitimité linguistique égale légitimité identitaire. Le fait de limiter la langue française à l'étude et de refuser de se conformer entièrement à la sphère française note d'une manière ou d'une autre une protection de son identité dans la mesure où ce pourcentage d'étudiants croit à certains préjugés s'ils l'utilisent dans un contexte communicationnel quotidien comme la dégradation de la langue d'origine, la perte des valeurs traditionnelles, le non-respect de son identité arabe.

Dans cette optique, abandonner le français dans leur échange quotidien est une preuve de leur appartenance identitaire et de l'influence majeure de la question identitaire sur la pratique

$\overline{\text { (Attitudes et représentations déclarées..)Dr. Racha Mohamed Mahmoud }}$ 
langagière. Le français, en tant que langue étrangère, constitue un moyen d'accès ou au moins de rapprochement à une sphère valorisée.

\subsection{Sécurité /insécurité linguistique}

En fait, "l'insécurité linguistique n'est pas tant finalement la compétence linguistique réelle des locuteurs, que les représentations qu'ils se font de cette compétence, la façon dont ils évaluent eux-mêmes cette compétence, et surtout la façon dont ils définissent en interaction, par rapport à la compétence, réelle et/ou fantasmée de leurs interlocuteurs"(Bretegnier, 1999:300). L'état de (in)sécurité linguistique dépend donc des paramètres de la situation de communication, ses partenaires, les rôles interactionnels, les relations entretenues entre eux et la norme qui s'impose dans le cadre de l'échange.

D'ailleurs, la représentation qu'ils ont de la norme et de leur propre discours pèse sur le type d'évaluation (estimer/sousestimer). "L'écart entre les représentations qu'ont les locuteurs sur leurs propres productions verbales et celles qu'ils ont sur le "bien parler"(Ibid:235) fait surgir l'insécurité linguistique. Tout dépend de leur perception, de leur conscience, de la présence de ce type de décalage entre usage légitime et usage effectif. Prendre conscience de la présence des instances normatives est acquis grâce à l'institution scolaire, l'université, lieu par définition d'apprentissage et par suite consacré à distinguer le bien parler du mal parler. Pourtant, la transgression à la norme prescrite n'est pas corrélative avec le sentiment d'insécurité linguistique, notamment, si ce décalage par rapport à la norme ne gêne pas le locuteur ou si le locuteur n'en est pas conscient. Cependant, cet acte de transgression relève parfois de la pure volonté du locuteur pour des visées d'humour, de provocation de son interlocuteur, pour la

$\overline{\text { (Attitudes et représentations déclarées..)Dr. Racha Mohamed Mahmoud }}$ 
transgression en soi-même ou pour imposer une nouvelle "norme".

En général, si un locuteur évalue sa façon de parler comme $\mathrm{A}$ et considère que la norme prescrite est $\mathrm{A}$, nous pouvons dire qu'il est en sécurité linguistique. Pourtant, s'il s'évalue comme B et prend A pour norme de prestige, il est en insécurité linguistique. Celle-ci se manifeste donc à travers le jugement négatif porté à sa façon de parler. L'insécurité linguistique signifie " écart entre les performances effectives des locuteurs, leurs jugements épilinguistiques et leurs représentations quant au bien parler"(Bretegnier, 1999:38) cet écart est révélateur d'insécurité linguistique. C'est un sentiment éprouvé par un locuteur "de ne pas se conformer à la norme socialement et situationnellement définie comme "légitime" et véhiculée par un groupe socioidentitaire auquel il n'appartient pas"(Ibid.). Ce sentiment se manifeste lorsqu'il parle ou croit qu'il parle mal. Il est à noter que la connaissance et la conscience de la norme de référence influence le degré d'insécurité linguistique

Leur perception de la communication en français est préférentiellement à des camarades $32 \%$ et en zone francophone $62 \%$. L'objectif communicationnel du français est diversifié. Leur choix quant à l'auto-évaluation de leurs compétences langagières (Q.5, Q.6, Q.8, Q.9, Q.14) permet d'examiner de près la notion de la sécurité et/ ou l'insécurité linguistique. Un taux très élevé de sécurité est notable grâce aux données chiffrées:

- l'attitude et l'évaluation positives, Q. 14, où72\% choisissent la variable de parler le français "couramment dans toutes les situations" et personne n'opte pour la réponse "insuffisamment dans toutes les situations", dans la Q. 8 où $56 \%$ pensent qu'ils parlent bien le français, le 
fait d'opter pour la réponse "bien parler français" correspond donc à une certaine confiance en soi ;

- la motivation stable de continuer à parler français, Q.51, pour $66 \%$ et dans Q.6, $76 \%$ sont satisfaits de leur pratique langagière.

D'ailleurs, le fait d'utiliser le français pour une finalité ludique montre un niveau moindre d'insécurité linguistique. La présence de la sécurité pourrait être l'effet de la scolarisation et de l'usage correspondant à la norme. Ils n'ont pas peur d'être socialement dépréciés puisque leur manière de parler est conforme aux enjeux de la situation de communication. Bien qu'il s'agisse d'une situation informelle, les locuteurs portent beaucoup d'attention à leur façon de parler. Leur pratique correspond à ce qu'ils pensent de bien parler. Leurs réalisations verbales, qu'ils jugent correctes ou non, c'est en fonction d'un modèle normatif à leur propre manière. C'est ainsi que dans la Q. 8, 30\% optent pour la variable "français à votre façon".

Pourtant un nombre assez limité manifeste une insécurité linguistique quant à l'usage du français dans un contexte communicationnel. Les étudiants fournissent dans Q.5 une réponse qui relève de l'insécurité: $56 \%$ pensent avoir un répertoire pauvre. Le fait de sous-estimer figure encore dans la Q. 9 où $30 \%$ trouvent qu'ils ont des difficultés à se comprendre, contre $18 \%$ qui sont certains de leur réussite dans l'échange. Nous jugeons que leur pratique est caractéristique d'insécurité linguistique lorsqu'"il $\mathrm{y}$ a un décalage entre leur usage personnel et l'usage qu'ils considèrent comme correct et conforme à la norme de référence, à titre d'exemple la Q. 19 montre que leur maîtrise n'est pas parfaite puisque le degré de spontanéité, pour $46 \%$, est limité et n'existe pas selon $42 \%$. L'écart entre une pratique beaucoup plus spontanée et leur propre pratique est un indice d'insécurité. 
L'usage approprié à une situation de communication donnée dénote qu'ils sont plutôt en sécurité linguistique. Nous constations quand même que les étudiants éprouvent une autre sorte d'insécurité, à savoir l'insécurité pour la langue. Il est à signaler que reconnaitre que la langue est en danger est un indice d'insécurité linguistique. Autrement dit, ce sentiment est ressenti pour le statut du français au Fayoum puisqu'il se trouve en situation de menace, de danger par rapport aux autres langues (l'anglais en premier et l'italien et l'espagnol en voie d'émergence).

\section{$\underline{\text { 3- Exemples de pratique }}$}

Il est à noter que les étudiants sont invités à la fin du questionnaire, si c'est possible, de fournir un exemple de leur propre pratique $\mathrm{du}$ français hors $\mathrm{du}$ contexte scolaire, avec n'importe qui et n'importe quand. Tester de près ces exemples nous permet de dire que ;

- Leur usage du français est rarement caractérisé par une forme normative. Nous remarquons une certaine rupture avec le français normé. Autrement dit, leur causerie montre l'écart entre ce qui correspond à la norme et ce qu'ils produisent réellement. Pourtant, les énoncés, dits normatifs, ressemblent à des clichés, à des énoncés tous faits, retenus par cœur. Le recours à ce type d'énoncés reflète un certain sentiment d'insécurité à l'égard de leur propre pratique. Ils considèrent leur niveau inférieur à la norme. Ils y attribuent donc une image moins légitime.

- L'analyse de ces productions souligne parfois un dilemme quant au choix de langage de démarrage. D'ailleurs, la variation du lieu de vie (milieu 
rural/urbain) pèse sur le choix du lexique soit arabe, soit français

- Ils optent très souvent pour l'alternance codique et les phrases inachevées. Les phrases sont également construites sur le modèle arabe.

- A certaines reprises, nous avons l'impression qu'ils tentent de mettre en scène un locuteur qui s'efforce d'adopter une pratique proche de celle du locuteur natif.

- Certains termes et expressions se réfèrent à l'éthos, revendiqué par soi-même ou attribué à l'autre : quelqu'un capable de communiquer en langue étrangère assure évidemment un certain prestige. Ils veulent ainsi manifester un ethos beaucoup plus distingué et distant par rapport à leurs pairs.

- La plupart du temps, ils se servent d'un syntagme introductif à une histoire, en français notamment, provoquant le suspens tels que "tu as vu /tu as su" sur le modèle arabe ( شوفني/ عرفني) ['rifti /Šufti]

- En matière des règles rituelles, la frontière tu/vous ne passe pas de la même manière qu'en français. De plus, la relation familière, les requêtes se font directement et parfois d'une manière assez brutale, sans recours aux adoucisseurs. Certaines répliques se répètent en écho: salutations, compliment de site, échange de vœu,...etc. Ce type d'acte de parole est donc régi par des obligations mutuelles. Certaines situations permettent plus de tolérance à l'affrontement direct, c'est pourquoi nous notons beaucoup d'interruptions. En plus, les formules conservant un caractère religieux soit de bénédiction, soit de malédiction (vœu/contre vœu) y

(Attitudes et représentations déclarées..)Dr. Racha Mohamed Mahmoud 
sont très fréquentes. En d'autres termes, beaucoup d'actes sont bénis et protégés ou au contraire repoussés et rejetés par Dieu comme cette structure :

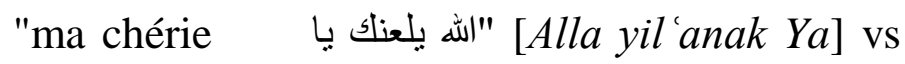

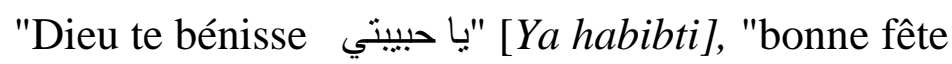
"يا جميل"[Ya Jamil]

- Leur pratique s'inscrit dans une pratique beaucoup plus large, à savoir le parler des jeunes. Ce type de parler comprend, si nous permettons de le dire, une sorte d'insulte rituelle. C'est un signe de l'appartenance et de l'intégration dans un groupe d'amis. L'absence de ce marqueur est même considéré comme un rejet, c'est-à-dire on n'est ni admis, ni connu dans un tel groupe. Elle reflète donc le degré de familiarité. Le profil communicatif de ce groupe d'étudiants se caractérise par le fonctionnement des termes d'adresse accompagnés des termes valorisants (جميل/حبيني) ou dévalorisants (les insultes associés à des animaux : noms ou attributs). Mais les deux formes d'adresse peuvent être décrites comme familières visant le pur accès au territoire privé : confidence et toute forme de parole intime. Donc les comportements langagiers sont constamment dissociables des comportements sociaux.

Alors, leurs productions sont en fonction de leurs propres normes aussi bien culturelles que langagières. Elles reflètent certaines présentations de soi, réelles ou feintes. Cette pratique montre une maîtrise relative du français et une capacité à communiquer malgré certaines erreurs. Ce groupe d'étudiants est

(Attitudes et représentations déclarées..)Dr. Racha Mohamed Mahmoud 
motivé parce qu'il trouve que le français constitue non seulement une langue d'étude mais plutôt un outil efficace de communication en dehors de la scolarité, ce qui exprime une attitude positive envers le français.

\section{Conclusion}

La pratique du français hors de l'université pourrait être envisagée différemment chez un locuteur essayant de légitimer un certain type d'usage du français coexistant avec l'arabe au plan social, culturel et identitaire. Chaque contexte appelle une façon de parler très particulière. Dans cette optique, celle -ci est considérée comme le modèle légitime. Ce dernier garantit un certain sentiment de sécurité chez ces étudiants quant à leur usage linguistique dans ce contexte. Ces étudiants sont dotés d'un certain "pouvoir" qui les rend aptes à modifier leur façon de parler pour s'approprier au contexte de l'échange. Le taux de sécurité est plus élevé. Les études qu'ils ont déjà faites confèrent un caractère de sécurité à leur façon de parler.

Certes, ces étudiants n'ont pas la même compétence linguistique. Pourtant, ils possèdent une attitude favorable quant au français. Ils parviennent à assurer une sorte d'intercompréhension. Malgré l'hétérogénéité au niveau de sexe, milieu social,..., ils sont décrits en tant que locuteurs francophones dans la mesure où ils manifestent les mêmes représentations en regard de cette langue: outil d'échange quotidien. L'analyse des données chiffrées donne à voir qu'ils se passionnent en parlant français. Ils ressentent un plaisir nonmotivé. Cette attitude positive envers le français dans la communication quotidienne assure une forte envie de la découverte d'un autre mode de vie. L'attitude favorable et la pratique qui en résulte font preuve d'une compétence linguistique

$\overline{\text { (Attitudes et représentations déclarées..)Dr. Racha Mohamed Mahmoud }}$ 
bilingue. Les règles du jeu social y sont mises en place. Leur attitude dépend de la situation de communication et de divers paramètres qui pourraient se modifier à tout moment. Leur pratique relève également de l'identité francophone en sauvegardant en même temps la langue maternelle. Le français dans leur communication quotidienne est donc un signe d'intégration et d'appartenance à une communauté beaucoup plus large, la communauté francophone. Le contact arabe/français paraît comme un cadre protecteur et sécurisant garantissant la compréhension réciproque, surtout dans les énoncés alternés.

D'après eux, parler est l'équivalent de s'approprier d'un style propre à leur entourage en fonction de leur conception et de leur représentation de l'acte de communication et ses partenaires. La pratique de la parole constitue donc non seulement un outil d'expression mais plutôt une sorte de pratique à la fois sociale et culturelle où divers procédés sont mis en œuvre afin de garantir la gestion de l'échange. Les relations interpersonnelles sont également mises en scène. La dynamique sociale donne à voir des pratiques langagières différenciées. Leur parler est conforme aux normes de cette tranche d'âge. Le contact des langues n'est qu'un engagement dans une voie interculturelle. D'ailleurs, ils réussissent à faire correspondre leur choix de code aux attentes de la situation de l'échange- comme s'il s'agissait d'un consensus préalable -afin de garantir la continuité de l'interaction. Alors, leur comportement linguistique est le reflet du milieu socioculturel auquel ils appartiennent. L'analyse démontre que cette tranche d'étudiants est plus au moins vigilante à leur parler à tel point que nous assistons à une évaluation du discours d'autrui ainsi qu'à une auto -évaluation par rapport à un modèle normatif, surtout dans le cas de l'emphase.

$\overline{\text { (Attitudes et représentations déclarées..)Dr. Racha Mohamed Mahmoud }}$ 
L'analyse de leurs réactions témoigne d'un attachement au français, d'une richesse langagière, d'une communication exolingue (passage d'une langue à l'autre), d'une reconnaissance réelle de leur pratique et d'un équilibre et d'un sauvetage de sa langue et culture d'origine. Pourtant, l'aménagement de ce bi/plurilinguisme prend plutôt la forme d'une simple juxtaposition qu'un véritable contact. Le rapprochement français/anglais est quasi-total. Ils sont tout à fait loin d'endiguer la motivation à communiquer en français. Leur situation linguistique, brossée à grands traits, favorise le français dans la sphère familiale malgré l'insécurité linguistique conçue pour quelqu'uns dans leur pratique. En gros, la pratique quotidienne de diverses langues suggère un certain rapport de force, même symbolique, entre ces langues.

Cette pratique est en premier lieu ludique. Cet aspect suggère un usage naturel du français, affirme un sentiment de légitimité en tant que francophone et montre une forte volonté d'avoir accès à un autre camp : social, linguistique et identitaire. L'aspect ludique développé au fil de leurs échanges reflète une certaine maîtrise linguistique, c'est ainsi qu'ils réussissent à connaitre les règles du jeu. Ils communiquent sur une modalité mixte interférentielle, oscillant entre trois langues. Ils s'amusent donc à faire des mélanges des langues. D'ailleurs, cet aspect pourrait être pris pour cryptique, auquel les autres ne peuvent pas avoir accès. Cette pratique affirme une certaine compétence linguistique qui leur permet de jouer avec les mots, pour ainsi dire: arabiser le français et franciser l'arabe. Ils réussissent à construire un modèle d'expression convenable aux besoins de cette jeune génération. Les comportements communicatifs s'incarnent dans une communauté beaucoup plus profonde, en l'occurrence la communauté des jeunes dont le profil communicatif se caractérise par le fonctionnement des termes d'adresse accompagnés des termes valorisants ou dévalorisants.

(Attitudes et représentations déclarées..)Dr. Racha Mohamed Mahmoud 
Chaque positionnement suscite un ensemble d'attitudes et de comportements sociolinguistiques et des répercussions au niveau des relations interpersonnelles. L'usage du français hors du contexte universitaire parait comme un masque de leur origine et une étiquette d'un statut social plus élevé. La divergence de leurs productions est due à la variété du système des valeurs de ce milieu. A plusieurs reprises, ils ne font que réaliser un calque purement pragmalinguistique. L'implication culturelle régit la pratique linguistique. L'insertion ou non des termes français dans leur échange quotidien est en rapport direct avec la définition identitaire et la conception de l'appartenance communautaire de ces étudiants. La prise de distance, pour certains étudiants, vis-àvis du français correspond à des raisons à la fois linguistique (l'incompétence) et identitaire (langue de soi-même/celle de l'autre) et encore communicationnelle (inadaptation à certains domaines énonciatifs). L'insécurité linguistique fait partie d'une autre insécurité plus large, à savoir l'insécurité socio-culturelle ou socio-identitaire. Mais l'insertion du français dans leur échange montre la reconnaissance d'une telle langue, d'une telle culture et le désir de l'employer dans des contextes énonciatifs variés hors du cadre universitaire.

Dans ce contexte, hors des études, leur modèle linguistique s'écarte de la norme prescriptive, imposée institutionnellement. C'est évident, c'est même une exigence d'un tel contexte. Ils optent pour une autre norme, dite subjective, convenable à tel usage. Il s'agit dons d'une question de choix. Nous assistons à une sorte de renversement de rapports de force, même symbolique entre l'usage légitime et leur modèle linguistique mis en place. Ces étudiants, quel que soit leur genre ou leur milieu social, réagissent selon certaines règles, implicitement ou explicitement et auxquelles ils confèrent un statut et un pouvoir bien déterminé 
en fonction de leurs représentations et leurs attitudes en regard de cette langue.

Bref, leurs attitudes dépendent d'énormes facteurs : le statut social proféré à cette langue, les facteurs affectifs et identitaires et l'intérêt de spontanéité sollicitée. L'alternance codique est plutôt un signe de solidarité à une telle communauté et une expression de leur identité. Leur réponse fait apparaître un sentiment de responsabilité pour le français. Leur souci est avant tout de parvenir à le sauvegarder permettant ainsi de s'inscrire dans la sphère francophone. Leur réponse porte donc à croire qu'ils prennent une attitude défensive plutôt qu'une attitude agressive. Nous y notons un certain positionnement projeté en fonction de l'image souhaitée $\mathrm{du}$ français dans leur environnement linguistique.

Nous parvenons à dire que ces étudiants réussissent à conférer à leur pratique langagière certaines étiquettes, à la fois sociales et linguistiques et que les attitudes linguistiques des étudiants sont inséparables de leur représentation sociale et de l'imaginaire linguistique qu'ils possèdent en regard de cette langue. Leurs attitudes reflètent un capital linguistique et idéologique possédant un pouvoir symbolique ou réel, conscient ou inconscient, spontané ou institutionnalisé. Le contact des langues semble assurer une certaine complémentarité. Leur façon de parler est un signe d'identification et de statut. Les traits linguistiques sont inséparables des traits relevant de la personnalité, du statut socioprofessionnel dans ce milieu circonscrit d'étudiants.

Pour conclure, la pratique langagière du français hors du contexte scolaire égale à

-conférer une valeur emblématique

(Attitudes et représentations déclarées..)Dr. Racha Mohamed Mahmoud 
-afficher une certaine étiquette sociale

-acquérir une nouvelle norme communicationnelle

-aider le locuteur à être reconnu comme un francophone effectif

-se marquer d'une certaine appartenance identitaire

-faire preuve d'une attitude valorisante et d'une moindre insécurité linguistique

-entraîner des sentiments positifs à son égard

-prouver une certaine représentation sociolinguistique

-revendiquer la légitimation d'un tel exercice

-manifester la liberté de choix du code

-être révélatrice de la présence de deux identités qui se construisent dans et par la langue

-ne pas remettre en question leur compétence linguistique en français

-assurer une double fonction : instrumentale (instrument de communication) et intégrante (intégrer dans une communauté sociolinguistique bien déterminée) 


\section{Bibliographie}

- Abric J., 2011, Pratiques sociales et représentation, Paris : Presses universitaires de France.

- Akinci M., 2006, "Un situation de contact de langues : le cas turcfrançais des immigrés turcs en France" in Contacts de langues, modèles, typologies, interventions, sous la direction de J.Billiez, Paris: L'Harmattan, pp. 127-144.

- Auger J., 1997, "Acquérir par l'enfant des normes sociolinguistiques" in M.L.Moreau (éd.), Sociolinguistique, concepts de base, Mardaga, pp.15-19.

- Billiez J., 2003, Sociologie du langage, Grenoble: Université Stendhal-Grenoble 3.

-Boyer H., 2003, De l'autre côté du discours: recherches sur les représentations communautaires, Paris: L'Harmattan.

-Bretegnier A., 1999, "Sécurité et insécurité linguistique. Approches sociolinguistique et pragmatique d'une situation de contacts de langues : la Réunion", thèse de doctorat, université de la Réunion.

- Calvet J., 2003, La sociolinguistique, Collection Que sais-je?, Paris: Presses universitaires de France.

1994, Les voix de la ville : introduction à la sociolinguistique urbaine, Paris: Payot.

- Dubois J., et al., 2012, Dictionnaire de linguistique et des sciences du langage, Paris: Larousse.

- Fioux P.et Tirvassen R., 1997, "Approches communicatives" in M.L. Moreau (éd.), Sociolinguistique, concepts de base, Mardaga, pp.47-49.

- Grawitz M., 1996, Méthodes des sciences sociales, Paris: Dalloz. 
-Houville S., 2012, Attitudes linguistiques: définitions, implications et application à l'anglais, Littératures, dumas-00931707 : htts://dumas.ccsd.cnrs.fr/dumas-00931707

- Lafontaine D., 1997, " Attitudes linguistiques", in M.L. Moreau (éd.), Sociolinguistique, concepts de base, Mardaga, pp.56-60.

1986, Le parti pris des mots. Normes et attitudes linguistiques, Bruxelles: Mardaga.

- Lopez J., 2006, "Alternance codique, normes sociales et clivage identitaire" in Contacts de langues, modèles, typologies, interventions, sous la direction de J.Billiez, Paris : L'Harmattan , pp.145-154

-Martinez P., 2011, La didactique de langues étrangères, Paris: Presses universitaires de France.

-Perret J., 2016, "Pratiques, attitudes et représentations linguistiques à Riverview, Nouveau-Brunswick", Travaux neuchâtelois de linguistique, n.64, pp.121-139.

- Pierozak I., 2006, "Contacts de langues sur internet: collisions/collusions? L'exemple des échanges en temps réel en français" in Contacts de langues, modèles, typologies, interventions, sous la direction de J.Billiez, Paris : L'Harmattan, pp.177- 190.

-Poutignat P., "Evènement de langue, situation sociolinguistique et interprétation" in Langage et société, n. 59, p.p. 47-72.

-Rispail M., 2006, "Contacts entre francique, français, allemande en Lorraine germanophone et au Luxembourg : situations/ phénomène/attitudes" in Contacts de langues, modèles, typologies, interventions, sous la direction de J. Billiez, Paris: L'Harmattan, pp. 79- 90.

- TREHEL N. et BLANCHET P., 2006, "Pratiques linguistiques régionales et représentations chez des élèves du primaire et de collège en zones suburbaines de Bretagne gallo" in Contacts de langues, modèles, typologies, interventions, sous la direction de J. Billiez, Paris: L'Harmattan, pp. 61-78. 
- Thiam N., 1997, " Alternance codique ", in M. L. Moreau (éd.), Sociolinguistique, concepts de base, Mardaga, pp.32-35.

-Thibault P., 1997, "Age", in M.L .Moreau (éd.), Sociolinguistique, concepts de base, Mardaga, pp.20-26.

- Wald P., 1997, "Choix de code" in M .L. Moreau (éd.), Sociolinguistique, concepts de base, Mardaga, pp.71-76. 


\section{$\underline{\text { Annexe (1) : Questionnaire }}$}

\section{Informations personnelles}

Nom :

Age:

Sexe: adresse (milieu rural/ urbain)

\section{Enseignement reçu (privé/public)}

Axe 1: la situation linguistique et le niveau de la maitrise du francais

1-Avec qui communiquer en français?

-entre pairs -entre les membres de familles -en zone francophone

2-Le taux de fréquence du français dans votre échange quotidien est ...

-énorme -moyen -minoritaire

3-La langue apprise en premier temps (l'anglais) parait ...

-Plus spontanée que le français

-plus élaborée que le français

-sur le même pied d'égalité que le français

4-La langue apprise en premier temps (l'anglais)....

-empêche la communication en français

-facilite la communication en français

-ne joue aucun rôle

5-Vous avez l'impression de posséder un répertoire ...

-riche et complexe $\quad$-pauvre $\quad$-difficile à évaluer

6-L'auto-évaluation de vos compétences langagières est ....

-positive -négative $\quad$-moyenne

(Attitudes et représentations déclarées..)Dr. Racha Mohamed Mahmoud 
7-L'acquisition de l'écrit .....

-est au service de l'oral -est loin de l'oral -persiste et la parole s'en vole 8-Vous pensez que vous parlez ...

-bien français - -mal français - français à votre façon

9-Vous éprouvez des difficultés à se comprendre...

$\begin{array}{lll}\text {-oui } & \text {-non } & \text {-on se sait pas }\end{array}$

10-Vous lisez des journaux en français.....

-fréquemment - parfois -non

11-Vous écrivez en français ....

$\begin{array}{lll}\text {-un conte } & \text {-un roman } & \text {-des articles }\end{array}$

12-Vous suivez les chaines de la télévision française ...

-fréquemment -rarement -non

13-Lors de votre communication en français, vous agissez ...

- en suivant les règles de la déontologie

- librement en préférant un discours non-formalisé

- -d'autres réponses

14-Vous parlez français....

-couramment dans toutes les situations

-différemment d'une situation à l'autre

-insuffisamment dans toutes les situations

15-Lors de la communication, vos problèmes linguistiques concernent....

-le choix du lexique approprié

-la structure grammaticale

-la prononciation correcte

(Attitudes et représentations déclarées..)Dr. Racha Mohamed Mahmoud 
16-Votre système graphique ..... -est diversifié $\quad$-est restreint $\quad$-se sert plutôt d'émoticônes

17-Votre emploi des rieurs est...
-massif
-minoritaire
-inexistant

18-Dans votre pratique langagière, vous ayez recours à des formules métalinguistiques et métacommunicatives

-oui -non $\quad$-pas de réponse

19-Le degré de spontanéité sollicitée ...

-est énorme $\quad$-est limité $\quad$-n'existe pas

20-Pendant votre échange, vous jouez sur les sonorités et les schémas intonatifs...

- Toujours - jamais $\quad$-parfois

21-Vous ayez recours à des énoncés normatifs .....

-rarement -fréquemment -jamais

\section{Axe 2: $\underline{\text { Contact/conflit des langues }}$}

22-Si la communication en français s'avère impossible, vous avez recours à ...

-l'arabe - l'anglais - d'autres langues

23-Le mélange des langues est d'ordre ....

-communicationnel -identitaire -acquisitionnel

24-Le parler bi/plurilingue assure une meilleure condition de la réussite de l'échange

-oui -non $\quad$-cela dépend

(Attitudes et représentations déclarées..)Dr. Racha Mohamed Mahmoud 
25-Le contact entre ces langues vous parait .....

-naturel -artificiel - exagéré

26-Les énoncés métissés ou alternés ....

-témoignent d'une certaine compétence bi/plurilingue

-évitent l'insécurité linguistique

-cherchent à légitimer une certaine pratique langagière

27-Les marques transcodiques concernent plutôt.....

-le plan syntaxique -le plan phonologique -le plan lexical

28-La communication en français est...

-symétrique (parler français et l'autre répond en français)

-dissymétrique (parler français et l'autre répond en une autre langue)

-mixte (parler français et l'autre répond en plusieurs langues)

29-L'articulation français/arabe correspond à ....

- un jeu - -un conflit - d'autres phénomènes

30-Le contact des langues vous aide à mieux se reconnaitre et communiquer

$\begin{array}{lll}\text {-oui } & \text {-non } & \text {-on ne sait pas }\end{array}$

\section{Axe 3 : Examiner directement les attitudes}

31-La langue étrangère dans laquelle vous vous sentez à l'aise au milieu familial est

-le français - l'anglais $\quad$-d'autres langues

32-Le choix de langue de la communication est déterminé par certains préjugés linguistiques...

-oui -non $\quad$-d'autres réponses

(Attitudes et représentations déclarées..)Dr. Racha Mohamed Mahmoud 
33-Votre comportement linguistique vis -à- vis du français est ...

-stable

-relativement modifié -entièrement modifié

34-La charge affective à l'égard du français est....

-forte -faible -modérée

35-Lorsque vous communiquez en français, vous ressentez ...

-fier et émouvant -honteux et dégradant - indifférent et insensible 36-La communication en français est ......

- Plus active -moins active -très monotone

37-Parler français est un parler .....

-valorisant -dévalorisant -neutre

38-Selon vous, le français vise à ....

-se faire des amis -accomplir des affaires -communiquer intra-famille 39-Vous utilisez le français pour ....

-discuter des sujets personnels

-aborder des sujets polémiques

-faire une plaisanterie

40-Le français est préférable dans un contexte.....

-ludique -cryptique -politique

41- Dans vos échanges, les fragments de plaisanterie en français sont

-énormes -minorés -inexistants

42-Dans votre production, la quelle se manifeste la plus......

-l'insulte rituelle - les pratiques ludiques - la transgression de normes (effet d'humour)

$\overline{\text { (Attitudes et représentations déclarées..)Dr. Racha Mohamed Mahmoud }}$ 
43-Vous communiquez en français pour ....

-témoigner de sa culture

-faire vivre cette langue dans votre milieu

-garder le contact avec des francophones

44-Votre pratique est conçue comme une forte volonté d'améliorer le degré de spontanéité et se familiariser avec le français...

-oui -non $\quad$-on ne sait pas

45-Echanger en français signifie se sentir .....

-marginalisé de toute communauté linguistique

-intégré dans une communauté francophone

-démarqué du groupe

46-Le taux du français dépend de la /du ....

-degré de formalité - charge affective -besoin communicatif

47-Le changement de l'interlocuteur impose un changement de la façon de parler

-oui -non $\quad$-pas de réponse

48-L'intercompréhension ou la réussite de la communication est revendiquée comme ...

-un atout -un besoin -un engagement

49-Les garanties de la réussite de l'acte de l'échange sont....

-relatives - absolues -inexistantes

50-Le français n'est pas une langue avec laquelle on passe une information mais plutôt une langue avec laquelle on nait, vous trouvez cette attitude ...

-exagérée - réelle - d'autres réponses

$\overline{\text { (Attitudes et représentations déclarées..)Dr. Racha Mohamed Mahmoud }}$ 
51-La motivation pour continuer à communiquer en français ...

-évolue -diminue $\quad$-demeure stable

52-Votre famille apparait à l'égard de votre pratique ....

-distante -motivée $\quad$-surprise

53-L'emphase avec laquelle, votre interlocuteur emploie souvent le français....

-vous irrite $\quad$-vous plait $\quad-$ ne vous touche pas

54-Face à ce comportement d'emphase, vous ...

-avez recours à la stratégie d'évitement

-réagissez sur le même plan

-lui faites la remarque (petit commentaire là-dessus)

55-Parler français est doté des traits ....

- sociaux - psychologiques - neurologiques

56-La pratique du français sert à promouvoir...

-plus de respect d'autrui -moins de respect d'autrui -d'autres réponses

57-Lorsque nous parlez français, vous éprouvez ....

-de la sécurité - -de l'insécurité -de la réticence

58-Dans votre pratique, les revendications identitaires...

-émergent clairement -émergent implicitement - ne se manifestent pas 59-S'éloigner du français normé sert à ....

-provoquer le rire -se libérer de l'univers scolaire -créer une rupture avec toute forme de règle

60-Communiquer en français est un signe de l'appartenance à ...

-la communauté des jeunes - la communauté francophone -les deux

$\overline{\text { (Attitudes et représentations déclarées..)Dr. Racha Mohamed Mahmoud }}$ 


\section{$\underline{\text { P.S. }}$}

Vous êtes invité, si c'est possible, à fournir un exemple de votre pratique du français hors du contexte universitaire, avec n'importe qui et n'importe quand : 


\section{الملخص}

تهدف هذه الدراسة إلى تحليل اتجاهات ومواقف استخدام اللغة الفرنسية في

سياق خارج إطار الجامعة باستخدام استبيان يضم ثلاثة محاور : الوضع اللغوي ومستوى إتقان الفرنسية، الاتصال والصراع اللغوي، الفحص المباشر لاتجاهات الطلاب. يتم تقديم هذا الاستبيان لعينة من خمسين طالبا ، في الغالب من الاناث، ناطقين بالفرنسية وموطنه الاصلي الفيوم. التحليل الاحصائي للبيانات الكمية يجعل من الممكن دراسة بعض الظواهر اللغوية الاجتماعية منل نوع الاتجاه ايجابي أو سلبي والمتغيرات الاجتماعية، الدوافع لاختيار الفرنسية كلغة اتصال، التتاوب اللغوي ووظائفه ، الامن وانعدام الامن اللغوي، الهوية اللغوية والاجتماعية. الطلاب مدعوون أيضا في نهاية الاستبيان لتقديم مثال لممارستهم اللغوية باللغة الفرنسية خارج الإطار الدراسي. الكلمات المفتاحية : الهوية اللغويةـ التناوب اللفوي- الاتصال والصراع اللغويالاتجاه 